\title{
DRAFT : Optimal Motion Generation for Exiting a Parallel Manipulator from a Type 2 Singularity
}

\author{
Georges Pagis $^{1,2,3}$, Sébastien Briot ${ }^{1 *}$, Nicolas Bouton $^{3}$ and Philippe Martinet ${ }^{1,2}$ \\ ${ }^{1}$ Institut de Recherche en Communications et Cybernétique de Nantes (IRCCyN), UMR CNRS 6597, 44321 Nantes, France \\ ${ }^{2}$ LUNAM, Ecole Centrale de Nantes, 44321 Nantes, France \\ ${ }^{3}$ Institut Pascal, IFMA, MMS department, UMR CNRS 6602, 63000 Clermont-Ferrand, France \\ \{Georges.Pagis,Sebastien.Briot,Philippe.Martinet\}@irccyn.ec-nantes.fr \\ nicolas.bouton@ifma.fr\}
}

June 26, 2019

\begin{abstract}
Parallel robots have proved they can have better performances than serial ones in term of rigidity and payload-to-weight ratio. Nevertheless their workspace is largely reduced by the presence of singularities. In particular, the Type 2 singularities (parallel singularities) separate the workspace in different aspects, corresponding to one (or more) robot assembly modes. In order to enlarge the workspace size, it has been proved that a mechanism can cross the singularity loci by using an optimal motion planning. However, if the trajectory is not robust to modeling errors, the robot can stop in the singularity and stay blocked.

Therefore, the objective of this paper is to show new general procedure that allows the exit of a parallel manipulator from a Type 2 singularity. Two strategies are presented. The first one proposes the computation of an optimal trajectory that makes it possible for the robot to exit the singularity. This trajectory must respect a criterion that ensures the consistency of the robot dynamic model all along the singularity loci. The second trajectory consists in declutching one of the robot actuator in order to change the kinematic and dynamic behavior of the mechanism so that no singularity exists anymore. Theoretical works are il-
\end{abstract}

\footnotetext{
*Address all correspondence to this author.
}

lustrated, discussed and analysed through simulations achieved on a planar five-bar mechanism.

\section{Introduction}

Parallel manipulators have many advantages in terms of acceleration capacities and payload-to-weight ratio, but one of their main drawbacks concerns the presence of singularities $[1,2,3,4,5]$ which divide the workspace into different aspects, each aspect corresponding to one (or more) assembly modes [6]. The physical interpretation of a singularity in kinematics refers to the parallel manipulators configurations in which either the manipulator loses the ability to move in some directions, or its platform gains instantaneously some unconstrained motions.

In [2], three different types of singularities are described:

- Type 1 singularities are configurations where the platform loses a degree of freedom; the Type 1 singularities are configurations where the endeffector reaches the limits of the workspace,

- Type 2 singularities are configurations where an uncontrollable motion of the platform appears; the Type 2 singularities have much more complex loci, which divides the workspace in differ- 
ent aspects, resulting in a reduction of the manipulator's workspace; in general, in the neighborhood of such singularities, the reaction in passive and active joints considerably increase, thus preventing the manipulator to cross them,

- Type 3 singularities are configurations where both Type 1 and Type 2 singular configurations appear at the same time.

Moreover, in addition of these first works, other types of singularities, such as the constraint singularities [5], have been studied. For a global overview of the singularity problem, the reader is referred to [3]. However, the present paper focus only on Type 2 singularities as they are probably the most constraining singularities a parallel manipulator can meet.

In order to increase the workspace size, largely reduced by Type 2 singularity, several approaches have been envisaged in the literature. The most usual one is the design of parallel robots without singularities. Many works have been proposed in this direction, using optimal design approaches [7, 8] or even the creation of decoupled mechanisms without any singularity $[9,10]$. However, except in the case of decoupled robot that have a lack of stiffness, the design of robots in which only a portion of the workspace is free of singularity is generally envisaged.

Another solution was to use redundancy $[11,12$, 13]. However, this solution is costly and increase the control complexity. To avoid this problem, it has been proposed in [4] to use mechanisms with variable actuation modes to increase the size of the workspace. These mechanisms allow, without the addition of any supplementary actuators, to change the way they are actuated, via the use of clutches. This low-cost solution is attractive, however, the change in the actuation mode must be done when the manipulator is stopped, thus increasing the time for doing the requested trajectory.

A last solution is to create mechanisms with singularities inside the workspace, but to plan trajectories that allow the manipulators to go from one assembly mode to another. Two types of approaches have been developed in this direction:

1. the non singular assembly mode changing [14,
15]: by encircling cusp points, it has been demonstrated that the robot assembly modes can be changed; however, the computation of the cusp point loci is still a challenge in kinematics and such solution requires high mathematical skills. Moreover, it does not guaranty that the manipulator will be able to reach the whole workspace aspects,

2. the singular assembly mode changing $[16,17,18]$ : using the manipulator inertial properties, it has been demonstrated that it was possible to cross a Type 2 singularity without any considerable increase in the joint reactions and input torques; in [16], the physical condition for crossing a Type 2 singularity has been demonstrated.

The last approach is promising. However, if the trajectory for crossing the singularity is poorly planned (because of problems of robustness to modeling errors, for example), there is a risk that the mechanism can stop on the singularity and stay blocked inside. If such case appears, some methodologies for exiting the singularities must be found. Therefore the present paper aims at defining two strategies for exiting a Type 2 singularity if the manipulator is stopped inside.

\section{Dynamic modelling of paral- lel mechanisms}

\subsection{Lagrangian formalism}

Let us consider a parallel manipulator composed of $m$ links, $n$ degrees of freedom (dof) and driven by $n$ actuators. In general, for such mechanism, the Lagrangian $L$ of the system is expressed as a function of both the actuator and platform coordinates, i.e.

$$
L=L(\mathbf{q}, \dot{\mathbf{q}}, \mathbf{x}, \mathbf{v})
$$

where

- $\mathbf{q}=\left[q_{1}, q_{2}, \ldots, q_{n}\right]^{T}$ and $\dot{\mathbf{q}}=\left[\dot{q}_{1}, \dot{q}_{2}, \ldots, \dot{q}_{n}\right]^{T}$ represent respectively the vector of active joints variables and active joints velocities, 
- $\mathbf{x}=[x, y, z, \phi, \psi, \theta]^{T}$ and $\mathbf{v}=[\dot{x}, \dot{y}, \dot{z}, \dot{\phi}, \dot{\psi}, \dot{\theta}]^{T}$ are the end-effector pose parameters and their derivatives with respect to time, respectively. $x$, $y$ and $z$ represent the position of the platform controlled point and $\phi, \psi$ and $\theta$ the orientation parameters of the platform about three axes $\mathbf{a}_{\phi}, \mathbf{a}_{\psi}$ and $\mathbf{a}_{\theta}$.

The Lagrangian dynamic formulation for the considered parallel manipulator can be thus expressed as [19],

$$
\tau=\frac{d}{d t}\left(\frac{\partial L}{\partial \dot{\mathbf{q}}}\right)-\frac{\partial L}{\partial \mathbf{q}}+\mathbf{B}^{T} \lambda
$$

where

- $\tau$ is the vector of the input efforts,

- $\lambda$ is the vector of the Lagrange multipliers, whose expression can be computed using

$$
\mathbf{A}^{T} \lambda=\frac{d}{d t}\left(\frac{\partial L}{\partial \mathbf{v}}\right)-\frac{\partial L}{\partial \mathbf{x}}=\mathbf{w}_{p}
$$

in which

- $\mathbf{A}$ and $\mathbf{B}$ are two matrices deduced from the kinematic model, such that $\mathbf{A v}=\mathbf{B} \dot{\mathbf{q}}[6]$,

- $\mathbf{w}_{p}$ is the wrench applied to the platform by the legs and external forces [20].

Then, by substituting (3) into (2), and assuming that the matrix $\mathbf{A}$ is regular, one can obtain [19]:

$$
\tau=\mathbf{w}_{b}+\mathbf{J}^{T}{ }^{0} \mathbf{w}_{p} \text {, in which } \mathbf{w}_{b}=\frac{d}{d t}\left(\frac{\partial L}{\partial \dot{\mathbf{q}}}\right)-\frac{\partial L}{\partial \mathbf{q}}
$$

where

- ${ }^{0} \mathbf{w}_{p}$ is the expression of the wrench $\mathbf{w}_{p}$ in the base frame, i.e. ${ }^{0} \mathbf{w}_{p}=\mathbf{D} \mathbf{w}_{p}$ with $\mathbf{D}$ the matrix relating the platform twist $\mathbf{t}$ (expressed in the base frame) to the vector $\mathbf{v}$ by $\mathbf{t}=\mathbf{D v}[6]$,

- $\mathbf{J}={ }^{0} \mathbf{A}^{-1} \mathbf{B}$ is the Jacobian matrix between the platform twist $\mathbf{t}$ and $\dot{\mathbf{q}}$, with ${ }^{0} \mathbf{A}$ is the expression of the matrix $\mathbf{A}$ in the base frame, i.e. ${ }^{0} \mathbf{A}=$ $\mathbf{A D}^{-1}$.

Thus, by analyzing (4), it can be deduced that if matrix ${ }^{0} \mathbf{A}$ is singular, the dynamic model (4) cannot be computed. As a consequence, the next part deals with the conditions of singularity for matrix ${ }^{0} \mathbf{A}$.

\subsection{Parallel mechanism's singularities}

As presented in the introduction, the authors of [2] present three different types of singularities that can be obtained through the analysis of the kinematic model:

$$
{ }^{0} \mathbf{A t}=\mathbf{B} \dot{\mathbf{q}}
$$

Type 1 singularities occur when the mechanism is in a position such as the kinematic matrix $\mathbf{B}$ becomes rank deficient. In such configurations, the mechanism loses the ability to move in one given direction.

Type 2 singularities occur when the kinematic matrix ${ }^{0} \mathbf{A}$ becomes rank deficient. The Type 2 singularities divides the workspace in different aspects, resulting in a reduction of the manipulator's workspace. Moreover, in the presence of such singularities, the robot may also not be able to resist to an external wrench applied on the platform and the reaction in joints grows to infinity.

Type 3 singularities are configurations where both Type 1 and Type 2 singular configurations appear at the same time. They are discarded in the following of the paper as they appear if both Types 1 and 2 singularity exist.

In the next section, it is explained how to pass through a Type 2 singularity without making the dynamic model (4) degenerating, thus keeping the joint reactions in the parallel manipulator finite.

\subsection{Condition for passing through a Type 2 singularity}

In [16], it has been proved that a parallel mechanism can cross a Type 2 singularity without making the dynamic model (4) degenerating if and only if the trajectory respects a particular criterion at the singular point. Indeed, on a Type 2 singularity, the columns of ${ }^{0} \mathbf{A}$ are linearly dependent, i.e. there exist a vector $\mathbf{t}_{s}$ such that:

$$
{ }^{0} \mathbf{A t}_{s}=0 \Leftrightarrow \mathbf{t}_{\mathbf{s}}{ }^{T}{ }^{0} \mathbf{A}^{T}=0
$$

The vector $\mathbf{t}_{s}$ represents the twist of the uncontrollable motion of the platform in the singularity locus 
[6]. Thus, multiplying (3) by $\mathbf{t}_{s}^{T}$ leads to :

$$
\mathbf{t}_{s}^{T}{ }^{0} \mathbf{A}^{T} \lambda=0 \Rightarrow \mathbf{t}_{s}^{T}{ }^{0} \mathbf{w}_{p}=0
$$

As a result, if the desired manipulator motion doesn't guaranty the creation of a wrench $\mathbf{w}_{p}$ that respects the condition (7), the dynamic model is degenerated and the manipulator desired input efforts must grow to infinity to produce the desired platform motion. Physically, this condition means that the parallel manipulator can cross the Type 2 singularity if and only if the wrench ${ }^{0} \mathbf{w}_{p}$ exerted by the legs and external efforts on the platform is reciprocal to the twist $\mathbf{t}_{\mathbf{s}}$ of the uncontrollable motion in the Type 2 singularity. Based on this observation, it has been proved in [16] that it is possible to plan a trajectory that crosses a Type 2 singularity as long as the criterion (7) is respected on the singularity. It should be finally mentioned that, for one given set of robot inertial parameters, this condition depends only on the input joint and platform coordinates, velocities and accelerations.

\section{Methodologies for exiting a singularity}

If any parallel manipulator user wants to cross a Type 2 singularity by applying the criterion (7), it should be aware that if the trajectory is poorly planned (because of problems of robustness to modeling errors, for example), the mechanism can stop on the singularity and stay blocked inside. If such case appears, some methodologies for exiting the singularities must be found. This part aims at defining two strategies for getting out of Type 2 singularities. The first one consists in generating an optimal motion for the mechanism in order to follow an exit trajectory that guaranties the input efforts continuity all along the path. The second solution proposed relies on declutching one of the motor in order to change the mechanism's dynamical behaviour.

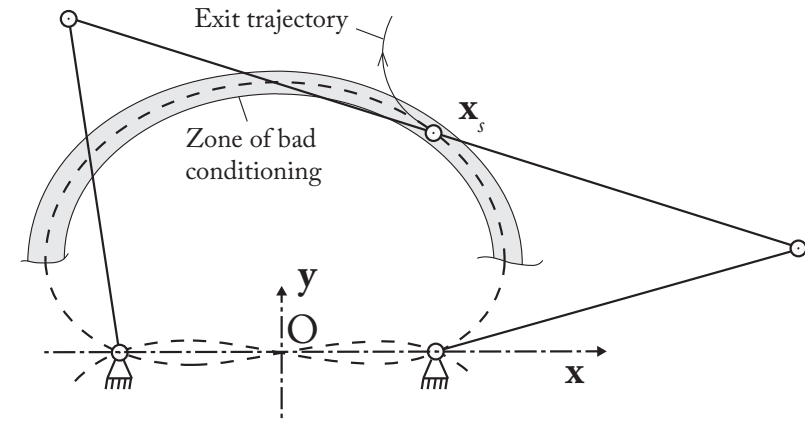

Figure 1: A PLANAR 5R MECHANISM BLOCKED ON A TYPE 2 SINGULARITY

\subsection{First solution: generation of an optimal motion}

The main idea of this solution can be explained as follows. Let us assume that the mechanism is stopped on the singularity locus at a point of coordinate $\mathbf{x}_{s}$ (as the 5R mechanism presented in Fig. 1). To exit the singularity, the mechanism must generate a trajectory that:

- is tangent to the singularity locus at point $\mathbf{x}_{s}$ as, when stopped, it cannot generate a motion ortogonal to the singularity locus,

- is $\mathcal{C}^{2}$ continuous to ensure the input effort continuity all along the path,

- respects the criterion (7) at point $\mathbf{x}_{s}$.

However, the mechanism being stopped into the singularity, i.e. its velocity being null when starting the motion at $\mathbf{x}_{s}$, if there is no external wrench applied such as the gravity field, this trajectory may fail exiting the robot from the singularity as it may have not enough energy to compensate the friction and local mechanical instability effects (due to the robot loss of stiffness) around the singularity point. As a result, in order to acquire enough energy, it is worth moving the robot inside the singularity loci during a certain amount of time before exiting the singularity. Thus, the trajectory can be cut into two parts:

1. a first trajectory that makes the robot platform move along the singularity locus, 
2. a second trajectory that is $\mathcal{C}^{2}$ continuous with the first one and able to make the robot go away from the singularity locus.

Obviously, the whole trajectory must guarantee that the input efforts remain finite during the whole motion. The way to compute these two different trajectories is detailed in the next sections.

\subsubsection{First trajectory: moving along the sin- gularity locus}

\section{Trajectory generation along the singularity}

In general, the computation of the singularity loci in the workspace is not an easy and straightforward problem [6]. Therefore, it is mostly difficult to get the symbolic constraint equations for the platform pose coordinates that impose the robot to stay in singularity. On the contrary, it is shown below that it is possible to find some sets of platform velocity parameters that can guaranty the robot to stay on the singularity loci.

Differentiating (6) with respect to time, it comes that:

$$
{ }^{0} \mathbf{A} \dot{\mathbf{t}}_{s}+{ }^{0} \dot{\mathbf{A}} \mathbf{t}_{s}=0
$$

in which, as matrix ${ }^{0} \mathbf{A}$ depends on the variables $\mathbf{x}$ and $\mathbf{q}$ only [6],

$$
{ }^{0} \dot{\mathbf{A}}=\sum_{i}\left(\frac{\partial^{0} \mathbf{A}}{\partial x_{i}} \dot{x}_{i}+\frac{\partial^{0} \mathbf{A}}{\partial q_{i}} \dot{q}_{i}\right)
$$

where $x_{i}\left(q_{i}\right.$, resp. $)$ is the $i$-th coordinate of the vector $\mathbf{x}$ (q, resp.). Multiplying the right side of (9) by $\mathbf{t}_{s}$, it comes:

$$
\begin{aligned}
{ }^{0} \dot{\mathbf{A}} \mathbf{t}_{s} & =\sum_{i}\left(\frac{\partial^{0} \mathbf{A}}{\partial x_{i}} \mathbf{t}_{s} \dot{x}_{i}+\frac{\partial^{0} \mathbf{A}}{\partial q_{i}} \mathbf{t}_{s} \dot{q}_{i}\right) \\
& =\mathbf{A}_{x}^{d} \mathbf{v}+\mathbf{A}_{q}^{d} \dot{\mathbf{q}}=\mathbf{A}_{x}^{d} \mathbf{D}^{-1} \mathbf{t}+\mathbf{A}_{q}^{d} \dot{\mathbf{q}}
\end{aligned}
$$

where the $i$-th column of $\mathbf{A}_{x}^{d}$ ( $\mathbf{A}_{q}^{d}$, resp.) is equal to $\frac{\partial^{0} \mathbf{A}}{\partial x_{i}} \mathbf{t}_{s}\left(\frac{\partial^{0} \mathbf{A}}{\partial q_{i}} \mathbf{t}_{s}\right.$, resp. $)$.

Introducing (5) into (10) leads to

$$
\begin{aligned}
{ }^{0} \dot{\mathbf{A}} \mathbf{t}_{s} & =\mathbf{A}_{x}^{d} \mathbf{D}^{-1} \mathbf{t}+\mathbf{A}_{q}^{d} \mathbf{B}^{-1}{ }^{0} \mathbf{A} \mathbf{t} \\
& =\left(\mathbf{A}_{x}^{d} \mathbf{D}^{-1}+\mathbf{A}_{q}^{d} \mathbf{B}^{-1}{ }^{0} \mathbf{A}\right) \mathbf{t} \\
& =\mathbf{A}_{x} \mathbf{t}
\end{aligned}
$$

Putting this expression into (8), it comes that

$$
{ }^{0} \mathbf{A} \dot{\mathbf{t}}_{s}+\mathbf{A}_{x} \mathbf{t}=0 \Rightarrow \mathbf{t}=-\mathbf{A}_{x}^{-1}{ }^{0} \mathbf{A} \dot{\mathbf{t}}_{s}=\mathbf{A}_{t} \dot{\mathbf{t}}_{s}
$$

As vectors $\mathbf{t}_{s}$ and $\dot{\mathbf{t}}_{s}$ are only defined on the singularity loci, (12) gives the conditions such that the robot stays in singularity. If the condition (12) is not verified for at least one value of the vector $\dot{\mathbf{t}}_{s}$, the manipulator could potentially exit the singularity.

As a result, to move the robot on the singularity, two conditions must be achieved:

- (12) for obtaining the kinematic constraints such that the robot can stay in singularity,

- (7) for obtaining the dynamic constraints that guarantee the dynamic model to be not degenerated.

Introducing (12) into (7) leads to the obtention of a nonlinear differential equation of the second order that must be solved for computing the robot displacement on the singularity loci.

\section{Computation of the input efforts}

Even if the criterion (7) is respected, it is still problematic to compute the input efforts when the robot is in the singularity. Having a look at (3), if $p$ denotes the rank of the matrix $\mathbf{A}$ in singularity $(p<n)$, it appears that only $p$ equations are linearly dependent. Thus, there is an infinity of possible sets for the values of the $n$ Lagrange multipliers $\lambda$ that satisfies the equation (3). Physically speaking, this means that the robot is locally over-constrained in the singularity. As a result, several approaches could be chosen to compute the values of the Lagrange multipliers such as the use of the pseudo-inverse of the matrix $\mathbf{A}$ (as it was done in the case of actuation redundant parallel robots [21]) or setting $(n-p)$ Lagrange multipliers to a given constant value. This last approach will be used in the following of the paper. 


\subsubsection{Second trajectory: exiting the singu- larity locus}

Trajectory generation for exiting the singularity

Once the mechanism has acquired enough energy, the second trajectory that allows the exit from the singularity loci must be followed. This trajectory must be $\mathcal{C}^{2}$ continuous with the first one in order to avoid any torque discontinuity (Fig. 1). Several types of $\mathcal{C}^{2}$ curves can thus be used, such as Lamé curves [22], clothoids [23, 24] or also S-curves [25]. In the following of the paper, clothoids are used. The way to link the clothoids to the trajectory defined in the section 3 is not detailed here. For more informations the reader is referred to [26].

One could wonder how to choose the aspect of the workspace in which the robot must go after exiting the singularity? Indeed, as on the singularity loci, one platform dof is not constrained, the robot is locally under-actuated. As a result, it seems impossible to plan a trajectory that is opposed to the free dynamics of this unconstrained dof. To know what is the best assembly mode to reach, the natural direction of motion of the mechanism free of actuation forces must be defined at the exit point. This can be done in simulation by declutching all actuators at the exit point after the motion along the singularity locus and by using the direct dynamic model that express the mechanism acceleration as a function of the input efforts (that are null in this case) to compute the robot displacement [19]. The mechanism will spontaneously move to one of the workspace aspects that will be the one to reach with the exit trajectory.

\section{Computation of the input efforts}

Theoretically, the matrix ${ }^{0} \mathbf{A}$ is not invertible only on the Type 2 singularity locus. However when the mechanism is very close to a singular position, the conditioning of the matrix ${ }^{0} \mathbf{A}$ is very high (Fig. 1). The dynamic model is therefore still degenerated in a area around the singularity locus. Inside this zone, it is irrelevant to use the numerical invert of ${ }^{0} \mathbf{A}$ to compute the input efforts. The best way to do is to consider that the robot is still in singularity in this area and to compute the input efforts using the methods presented in the Section 3.1.1.

When the robot exits this zone, i.e. when the conditioning of the matrix ${ }^{0} \mathbf{A}$ becomes acceptable, the input efforts can be computed using (2) and (3). In the following of the paper, the value of the conditioning of the matrix ${ }^{0} \mathbf{A}$ is said acceptable if it is equal or inferior to 100. Obviously, this value is arbitrary, but it will be shown in the next section that it leads to correct results.

\subsubsection{Discussion on the amount of energy necessary for exiting the singularity}

It has been explained above that the change between the two trajectories must be achieved when the mechanism has acquired enough energy to exit the singularity. So, when does this appear and how to have the corresponding information? This point is still an open question for the moment, however, one possible answer is to consider the energy consumpted by the actuators for compensating the friction in the passive joints and the energy of the moving robot platform and passive links. If the amount of the robot platform and passive links energy is large enough (e.g. 5 times larger) with respect to the amount of energy for compensating the dissipative terms, thus the change on the trajectory can be done. This point must be deeply analyzed in our future work.

\subsection{Second solution: declutch one of the actuators}

A second solution for exiting the singularity is to change the robot dynamics by changing its actuation mode, e.g. by declutching one of the actuators from the rest of the robot. As a consequence, the parallel mechanism becomes underactuated, which completely changes its singular configurations and dynamic properties.

Without loss of generality, let us assume that the input effort of the actuator $n$ is vanished, i.e. the last component of vector $\tau$ of (2) is set to 0 . Rewritting 
(2), it comes that:

$$
\left[\begin{array}{c}
\tau_{1} \\
\vdots \\
\tau_{n-1} \\
0
\end{array}\right]=\left[\begin{array}{c}
w_{b_{1}} \\
\vdots \\
w_{b_{n-1}} \\
w_{b_{n}}
\end{array}\right]+\left[\begin{array}{c}
\mathbf{b}_{1} \\
\vdots \\
\mathbf{b}_{n-1} \\
\mathbf{b}_{n}
\end{array}\right] \lambda, \mathbf{w}_{p}=\left[\begin{array}{c}
\mathbf{a}_{1} \\
\vdots \\
\mathbf{a}_{n-1} \\
\mathbf{a}_{n}
\end{array}\right] \lambda
$$

where $\tau_{i}$ is the $i$-th component of the vector $\tau, w_{b_{i}}$ the $i$-th component of the vector $\mathbf{w}_{b}, \lambda_{i}$ is the $i$-th component of the vector $\lambda, \mathbf{a}_{i}$ the $i$-th row of the matrix $\mathbf{A}^{T}$ and $\mathbf{b}_{i}$ the $i$-th row of the matrix $\mathbf{B}^{T}$. Rearranging these equations,

$$
\begin{aligned}
& {\left[\begin{array}{c}
\tau_{1} \\
\vdots \\
\tau_{n-1}
\end{array}\right]=\left[\begin{array}{c}
w_{b_{1}} \\
\vdots \\
w_{b_{n-1}}
\end{array}\right]+\left[\begin{array}{c}
\mathbf{b}_{1} \\
\vdots \\
\mathbf{b}_{n-1}
\end{array}\right] \lambda} \\
& {\left[\begin{array}{c}
\mathbf{w}_{p} \\
-w_{b_{n}}
\end{array}\right]=\left[\begin{array}{c}
\mathbf{a}_{1} \\
\vdots \\
\mathbf{a}_{n-1} \\
\mathbf{a}_{n} \\
\mathbf{b}_{n}
\end{array}\right] \lambda=\mathbf{A}^{*} \lambda}
\end{aligned}
$$

in which the matrix $\mathbf{A}^{*}$ is now invertible and can be used to compute the Lagrange multipliers in the singularity loci. Obviously, the expression (15) must be used on the singularity only (and also in a small zone around, as explained in the Section 3.1.2). As soon as the robot is nomore on the singularity, matrix $\mathbf{A}$ becomes regular and (13) can be used as it. However, as one actuator is declutched and as the robot is under-actuated, the direct dynamic model must be used for computing the robot displacement and being sure that there is no link collision during the motion [19]. In order to avoid this problem, the best way to proceed is to give a small impulse of motion to only one of the actuators and to stop it quite quickly in order to avoid a too large platform displacement.

\subsection{Discussion}

The two proposed solutions have advantages and drawbacks. In the first solution, the mechanism is fully controlled during its whole motion while in the second one, as one actuator is declutched, there is

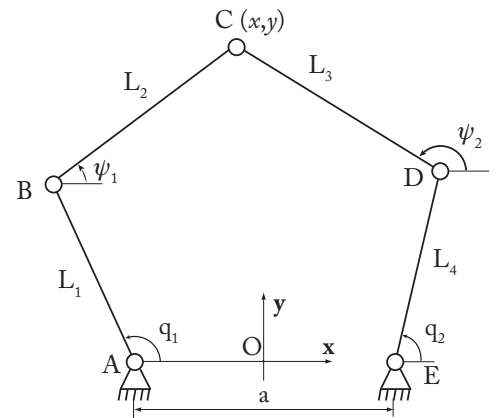

Figure 2: PLANAR 5R PARALLEL MECHANISM DESCRIPTION

a risk that the robot falls down under the application of external efforts such as the gravity. However, the first solution requires larger computational skills and needs to define two special types of trajectories whereas the second one seems simpler in terms of computation complexity. Therefore, both solutions have interests, depending of robots on which they are applied.

Another question concerns the fact that, in reality, the mechanism will never be exactly stopped on the singularity locus. However, it can be decided that, if the determinant of the matrix $\mathbf{A}$ is greater than a given value, the eigenvector of $\mathbf{A}$ corresponding to the smallest eigenvalue is equal to the twist $\mathbf{t}_{s}$ and all derivations can be achieved using this assumption.

\section{Illustrative example}

The two methodologies proposed to exit a Type 2 singularity are applied and simulated using the model of a $5 R$ planar mechanism.

\subsection{Description and modelling of the 5R mechanism}

The planar $5 R$ parallel mechanism is composed of two legs connecting the end-effector located at point $\mathrm{C}$ to the base by a revolute joint, as shown in Fig. 2. Each leg is composed of two rigid elements, called the proximal and distal elements, respectively. The 


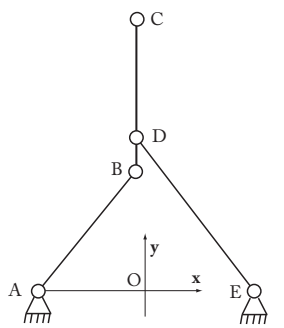

(a) $\psi_{1}=\psi 2$

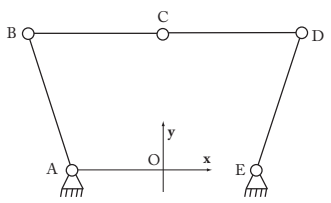

(b) $\psi_{1}=\psi 2+\pi$
Figure 3: TYPE 2 SINGULARITIES OF THE 5R PLANAR MECHANISM.

proximal element is linked to the base via an actuated revolute joint and to the proximal element via a passive revolute joint. The two revolute joints connecting the legs to the base are actuated (located at the points $\mathrm{A}$ and $\mathrm{E}$ on the Fig. 2), which enables the end-effector located at point $\mathrm{C}$ to be positionned in the plane $(O x y)$.

The notations used in the following of the paper are:

- $q_{1}$ and $q_{2}$ are the angles of the actuated revolute joints

- $\psi_{1}$ and $\psi_{2}$ are the passive angles of joints located at points $\mathrm{B}$ and $\mathrm{D}$

- $L_{i}$ is the length of the leg $i$

- $x$ and $y$ are the end-effector's positions along the $\mathbf{x}$ and $\mathbf{y}$ axes, respectively,

- $a$ is the distance between the two actuators.

For the $5 R$ planar mechanism, Type 2 singularities occur when the two distal elements are parallel [27]. Two different cases may appear, as shown on the Fig. 3, either $\psi_{1}=\psi_{2}$ (Fig. 3(a)) or $\psi_{1}=\psi_{2}+\pi$ (Fig. $3(\mathrm{~b}))$.

In the following of the paper, the gravity field is directed along the $\mathbf{z}$-axis. The equations of the dynamic model for the $5 R$ mechanisms have been described in [16], and the vectors and matrices necessary for com-

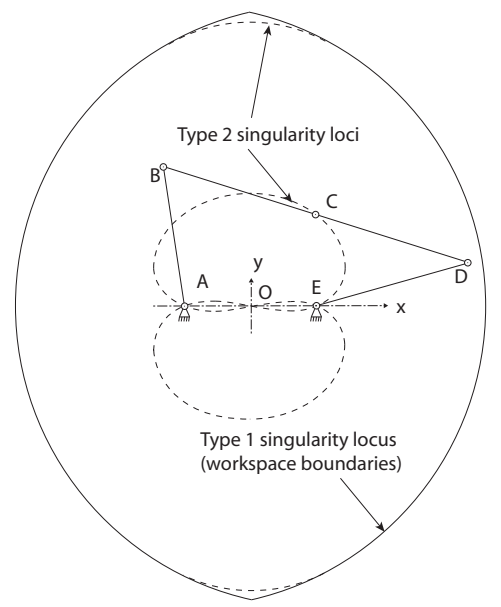

Figure 4: SINGULARITY LOCI FOR THE PLANAR 5R MECHANISM UNDER STUDY.

puting the dynamic model (2) take the form:

$$
\begin{aligned}
\mathbf{w}_{b} & =\left[\begin{array}{l}
m_{B_{1}} L_{1}^{2} \ddot{q_{1}}+m_{C_{1}} L_{1}\left(\ddot{y} \cos q_{1}-\ddot{x} \sin q_{1}\right) \\
m_{D_{2}} L_{4}^{2} \ddot{q}_{2}+m_{C_{3}} L_{4}\left(\ddot{y} \cos q_{2}-\ddot{x} \sin q_{2}\right)
\end{array}\right] \\
\mathbf{w}_{p} & =\left[\begin{array}{l}
m_{C_{2}} \ddot{x}-m_{C_{1}} L_{1}\left(\ddot{q}_{1} \sin q_{1}+\dot{q}_{1}{ }^{2} \cos q_{1}\right) \\
m_{C_{2}} \ddot{y}+m_{C_{1}} L_{1}\left(\ddot{q}_{1} \cos q_{1}-\dot{q}_{1}{ }^{2} \sin q_{1}\right)
\end{array}\right] \\
& +\left[\begin{array}{l}
-m_{C_{3}} L_{4}\left(\ddot{q}_{2} \sin q_{2}+\dot{q}_{2}^{2} \cos q_{2}\right) \\
m_{C_{3}} L_{4}\left(\ddot{q}_{2} \cos q_{2}-\dot{q}_{2}{ }^{2} \sin q_{2}\right)
\end{array}\right]
\end{aligned}
$$

$\mathbf{A}=\left[\begin{array}{ll}x+a / 2-L_{1} \cos q_{1} & y-L_{1} \sin q_{1} \\ x-a / 2-L_{4} \cos q_{2} & y-L_{4} \sin q_{2}\end{array}\right]=\left[\begin{array}{ll}a_{11} & a_{12} \\ a_{21} & a_{22}\end{array}\right]$

$\mathbf{B}=-\left[\begin{array}{cc}L_{1}\left(\sin q_{1} a_{11}-\cos q_{1} a_{12}\right) & 0 \\ 0 & L_{4}\left(\sin q_{2} a_{21}-\cos q_{2} a_{22}\right)\end{array}\right]$

with

$$
\begin{aligned}
& {\left[\begin{array}{l}
\dot{q}_{1} \\
\dot{q}_{2}
\end{array}\right]=\mathbf{B}^{-1} \mathbf{A}\left[\begin{array}{c}
\dot{x} \\
\dot{y}
\end{array}\right]} \\
& {\left[\begin{array}{l}
\ddot{q}_{1} \\
\ddot{q}_{2}
\end{array}\right]=\mathbf{B}^{-1}\left(\mathbf{A}\left[\begin{array}{l}
\ddot{x} \\
\ddot{y}
\end{array}\right]+\dot{\mathbf{A}}\left[\begin{array}{c}
\dot{x} \\
\dot{y}
\end{array}\right]-\dot{\mathbf{B}}\left[\begin{array}{l}
\dot{q}_{1} \\
\dot{q}_{2}
\end{array}\right]\right)}
\end{aligned}
$$


and

$$
\begin{aligned}
& m_{C_{1}}=m_{22} r_{2}\left(1-r_{2}\right) \\
& m_{C_{2}}=m_{22} r_{2}^{2}+m_{32} r_{3}^{2}+m_{23}+m_{21} \\
& m_{C_{3}}=m_{32} r_{3}\left(1-r_{3}\right) \\
& m_{B_{1}}=m_{12} r_{1}^{2}+m_{22}\left(1-r_{2}\right)^{2}+m_{13}+m_{21} \\
& m_{D_{2}}=m_{32}\left(1-r_{3}\right)^{2}+m_{42} r_{4}^{2}+m_{31}+m_{41}
\end{aligned}
$$

in which $r_{1}\left(r_{2}, r_{3}, r_{4}\right.$, resp.) is a ratio such that $r_{1} L_{1}\left(r_{2} L_{2}, r_{3} L_{3}, r_{4} L_{4}\right.$, resp. $)$ is the distance between the center of masses of the element $1(2,3,4$, resp.) and the point $\mathrm{A}$ ( $\mathrm{B}, \mathrm{D}, \mathrm{E}$, resp.), and

$$
\left[\begin{array}{l}
m_{j 1} \\
m_{j 2} \\
m_{j 3}
\end{array}\right]=\left[\begin{array}{ccc}
1 & 1 & 1 \\
r_{j} & 0 & 1-r_{j} \\
r_{j}^{2} L_{j}^{2} & 0 & \left(1-r_{j}\right)^{2} L_{j}^{2}
\end{array}\right]^{-1}\left[\begin{array}{c}
m_{j} \\
0 \\
I_{j}
\end{array}\right] \text {, for } j=1,
$$

where $m_{j}$ is the mass of the link $j$ and $I_{j}$ its axial moment of inertia expressed at the center of masses (for $j=1, \ldots, 4$ ). For deeper explanations on the way to obtain these expressions, the reader is referred to [16].

In the following of the paper, the values of the geometric and inertial parameters of the robot are set to:

- $L_{1}=L_{2}=L_{3}=L_{4}=0.25 \mathrm{~m}$ and $\mathrm{a}=0.2 \mathrm{~m}$

- $m_{1}=m_{4}=2.81 \mathrm{~kg}$

- $m_{2}=m_{3}=1.41 \mathrm{~kg}$

- $I_{1}=I_{4}=0.02 \mathrm{~kg} \cdot \mathrm{m}^{2}$

- $I_{2}=I_{3}=0.01 \mathrm{~kg} \cdot \mathrm{m}^{2}$

\subsection{Moving a $5 \mathrm{R}$ mechanism out of a singular position}

Let us now assume that the $5 R$ mechanism is blocked on the singularity, such as represented in Fig. 4. In this example, the end-effector position is set at: $\mathbf{x}_{0}=[0.13 m, 0.12 m]^{T}$. This section presents the calculation of the exit trajectory computed using the two methodologies.

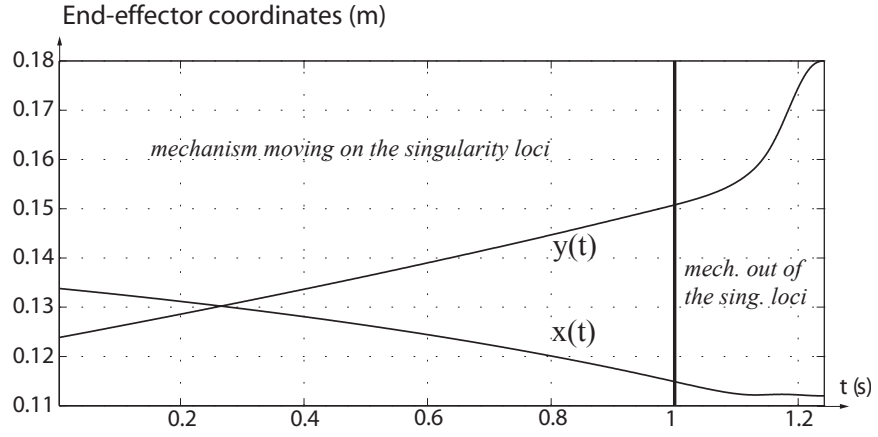

Figure 5: EVOLUTION OF THE END EFFECTOR COORDINATES ALONG THE EXIT TRAJECTORY

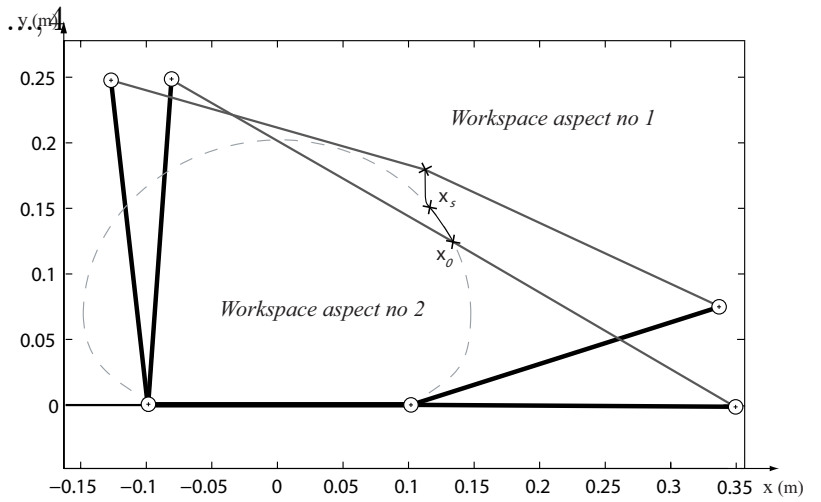

Figure 6: EXIT TRAJECTORY OF THE 5R MECHANISM

\subsubsection{First Solution: generation of an opti- mal motion}

Moving along the singularity locus

In order to move along the singularity, it has been shown in Section 3 that the two conditions (7) and (12) must be respected. For the studied robot, the vector $\mathbf{t}_{s}$ that defined the uncontrollable twist of the robot in the Type 2 singularity is equal to $[-\sin \psi, \cos \psi]^{T}[16]$, where

$$
\psi=\tan ^{-1}\left(\frac{y-L_{1} \sin q_{1}}{x-L_{1} \cos q_{1}-a / 2}\right)
$$




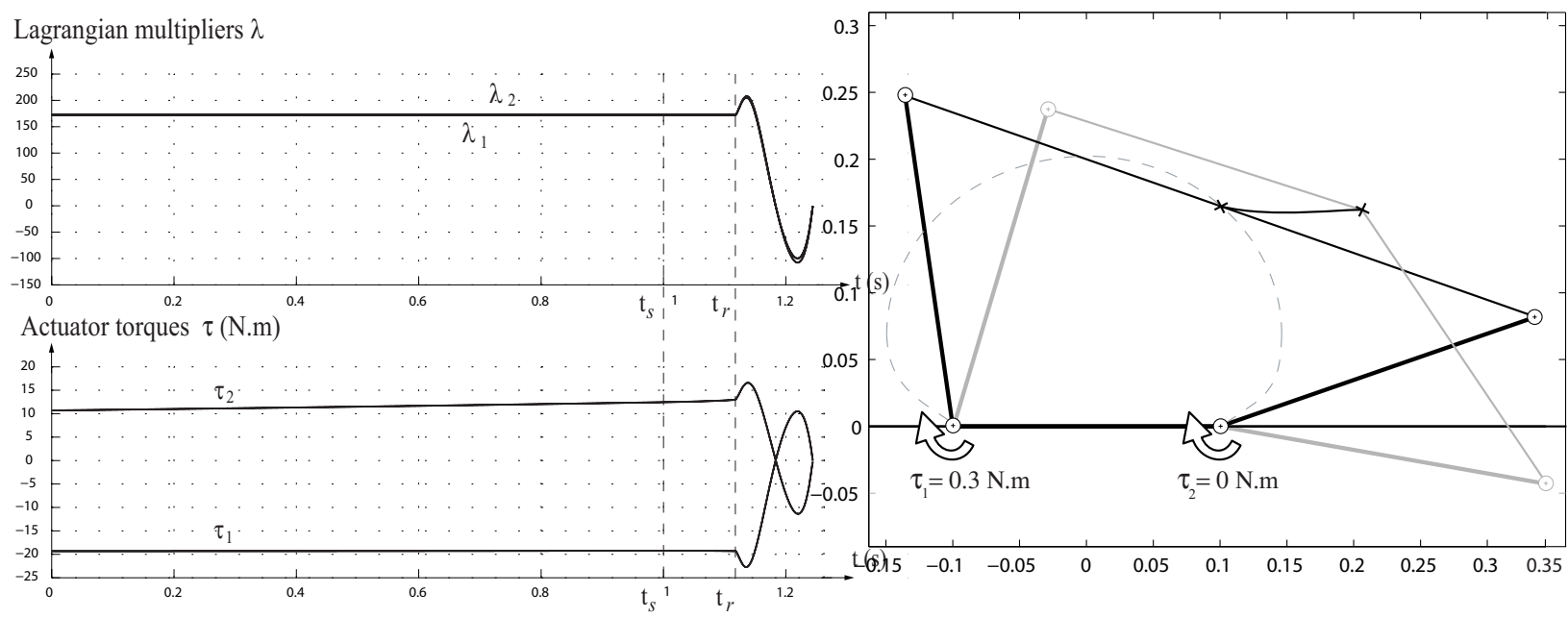

Figure 7: TORQUES VALUES ON THE EXIT

Figure 8: EXIT TRAJECTORY WITH THE SECTRAJECTORY

Thus, condition (7) can be expressed as:

$$
\begin{gathered}
-\sin \psi\left[m_{C_{2}} \ddot{x}-m_{C_{1}} L_{1}\left(\ddot{q_{1}} \sin q_{1}+\dot{q}_{1}^{2} \cos q_{1}\right)\right. \\
\left.-m_{C_{3}} L_{4}\left(\ddot{q}_{2} \sin q_{2}+\dot{q}_{2}^{2} \cos q_{2}\right)\right] \\
+\cos \psi\left[m_{C_{2}} \ddot{y}+m_{C_{1}} L_{1}\left(\ddot{q_{1}} \cos q_{1}-\dot{q}_{1}^{2} \sin q_{1}\right)\right. \\
\left.+m_{C_{3}} L_{4}\left(\ddot{q}_{2} \cos q_{2}-\dot{q}_{2}{ }^{2} \sin q_{2}\right)\right]=0
\end{gathered}
$$

in which the values of $\dot{q}_{i}$ and $\ddot{q}_{i}$ are expressed in (20).

Moreover, developping condition (12), it comes that:

$$
\left[\begin{array}{c}
\dot{x} \\
\dot{y}
\end{array}\right]=\mathbf{A}_{x}^{-1} \mathbf{A}\left[\begin{array}{cc}
\dot{\psi} & \cos \psi \\
\dot{\psi} & \sin \psi
\end{array}\right] \text { where } \mathbf{A}_{x}=\mathbf{A}_{x}^{d}+\mathbf{A}_{q}^{d} \mathbf{A}
$$

$\mathbf{A}_{x}^{d}=\left[\begin{array}{ll}-\sin \psi & \cos \psi \\ -\sin \psi & \cos \psi\end{array}\right], \mathbf{A}_{q}^{d}=-\left[\begin{array}{c}L_{1} \cos \left(\psi-q_{1}\right) \\ 0\end{array}\right.$ of bad conditionning of matrix $\mathbf{A}$ ). The input torques

Starting with $\dot{x}=-0.0127 \mathrm{~m} / \mathrm{s}$ and $\dot{y}=0.0124 \mathrm{~m} / \mathrm{s}$, the non-linear differential equations are solved using Matlab toolboxes for a trajectory duration of $1 \mathrm{~s}$. The resulting values are plotted in Fig. 5 for $t \leq 1 \mathrm{~s}$.

After $1 \mathrm{~s}$ of motion, the mechanism is in the position $\mathbf{x}_{s}=[0.11,0.15]^{T}$ (Fig. 6), with a velocity of 0.26 $\mathrm{m} / \mathrm{s}$ and an acceleration of $0.44 \mathrm{~m} / \mathrm{s}^{2}$. To know in which workspace aspect the mechanism should exit, its free behavior is simulated, i.e. the actuators are virtually suppressed in simulation at $t=1 \mathrm{~s}$. In the studied case, the mechanism is spontaneously moving to workspace aspect numbered as the aspect 1 in the Fig. 6 .

It can be demonstrated that the radius of curvature of the singularity locus at $\mathbf{x}_{s}$ is equal to $R_{c}=0.137 \mathrm{~m}$, which permits to define the clothoid that can extend the exit trajectory after $t=1 \mathrm{~s}$. The values of the end-effector position when moving along the clothoid path are presented in Fig. 5 when $t>1$ s. The total trajectory in the workspace is shown in Fig. 6 .

Using the methodology presented in section 3.1, the input torques are computed with a constant value for the Lagrange multiplier $\lambda_{2}=\lambda_{2}\left(X_{r}\right)=1.72$ till the mechanism is on the singularity (or in the zone the input torques are completely finite on the whole trajectory, even when moving on the singularity loci.

\subsubsection{Second Solution: Declutch one of the actuators}

In this section the mechanism is stopped in the same singular position as in Section 4.2.1. 


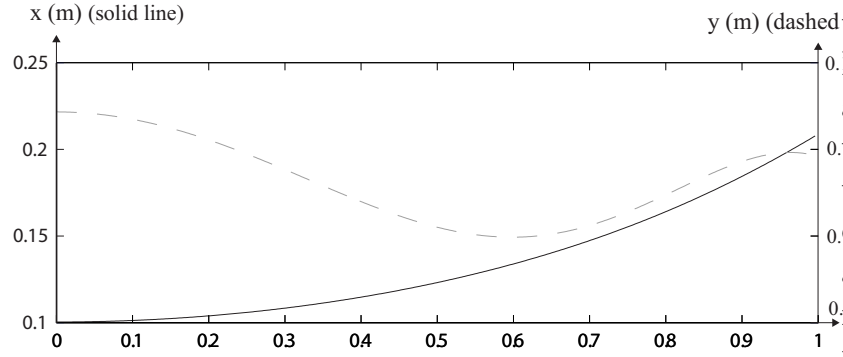

Figure 9: EVOLUTION OF THE END EFFECTOR COORDINATES ALONG THE DECLUTCHED TRAJECTORY

The second actuator is declutched and a constant torque equal to $0.3 \mathrm{~N}$.m is applied on the first actuator. Using the equations defined in section 3.2, the mechanism displacement can be computed. After $250 \mathrm{~ms}$, the mechanism is out of the area where the condition number of the matrix $\mathbf{A}$ is superior to 100. Figure 8 represents the computed exit trajectory in the mechanism workspace. It can be shown that the mechanism exit the singularity in the workspace aspect number 1 . The end effector coordinates along this trajectory are represented on Figure 9.

It could also be shown that by applying on motor 1 the opposite torque, the mechanism exit the singularity in the other workspace aspect (number 2). Based on the initial singular position, the input torques can be adjusted in order to move the mechanism in the desired assembly mode.

\section{Conclusion}

In order to increase the workspace size, past works have proved that a mechanism can cross the singularity loci by using an optimal motion planning. However, if the trajectory is not robust to modelling errors, the robot can stop in the singularity and stay blocked. Therefore, this paper proposes a new generic method dedicated to parallel manipulators in order to allow the exit from a Type 2 singularity. Based on the robot dynamic model, two strategies have been presented. The first one consists in a new algorithm dedicated to the computation of an optimal trajec- dtiogy that allows the robot to exit the singularity. 0.1610re precisely, the optimal trajectory is computed according to a specific dynamic criterion that ensures o.thye consistency of the robot dynamic model all along the singularity loci. Then, the second trajectory is o.characterized by the declutching of one of the robot actuator in order to change the kinematic and dyortamic behavior of the mechanism. Therefore, the und position, and its dynamic model is used to move away from the original mechanism Type 2 singularity.

Theoretical developments are illustrated, discussed and analysed through advanced simulations achieved on a planar five-bar mechanism. Simulations results demonstrate that the proposed algorithms permit to exit the parallel manipulator from the singularity. Furthermore, the optimal trajectory planning guarantee that the mechanism input torques stay finite even when the mechanism is moving on a singularity.

Future works will be dedicated to the implementation of the proposed algorithms on a real five-bar mechanism that is currently being developed.

\section{References}

[1] Bonev, I., Zlatanov, D., and Gosselin, C., 2003. "Singularity analysis of 3-dof planar parallel mechanisms via screw theory". ASME Journal of Mechanical Design(3).

[2] Gosselin, C., and Angeles, J., 1990. "Singularity analysis of closed-loop kinematic chains". IEEE Transactions on Robotics and Automation, $6(3)$, pp. 281-290.

[3] Conconi, M., and Carricato, M., 2009. "A new assessment of singularities of parallel kinematic chains". IEEE Transactions on Robotics, 25(4), pp. $757-770$.

[4] Arakelian, V., Briot, S., and Glazunov, V., 2008. "Increase of singularity-free zones in the workspace of parallel manipulators using mechanisms of variable structure". Mechanism and Machine Theory. 
[5] Zlatanov, D., Bonev, I., and Gosselin, C., 2002. "Constraint singularities of parallel mechanisms". In Proceedings of the IEEE International Conference on Robotics and Automation (ICRA 2002).

[6] Merlet, J., 2006. Parallel Robots, 2nd ed. Springer.

[7] Briot, S., Pashkevich, A., and Chablat, D., 2010. "Optimal technology-oriented design of parallel robots for high-speed machining applications". In Proceedings of the 2010 IEEE International Conference on Robotics and Automation (ICRA 2010).

[8] Liu, X.-J., Wang, J., and Pritschow, G., 2006. "Performance atlases and optimum design of planar $5 \mathrm{r}$ symmetrical parallel mechanisms". Mechanism and Machine Theory, 41(2), pp. 119-144.

[9] Kong, X., and Gosselin, C., 2002. "A class of 3-dof translational parallel manipulators with linear input-output equations". In Proceedings of the Workshop on Fundamental Issues and Future Research Directions for Parallel Mechanisms and Manipulators, pp. 3-4.

[10] Gogu, G., 2004. "Structural synthesis of fullyisotropic translational parallel robots via theory of linear transformations". European Journal of Mechanics. A/Solids.

[11] Kurtz, R., and Hayward, V., 1992. "Multiplegoal kinematic optimization of a parallel spherical mechanism with actuator redundancy". IEEE Transactions on Robotics and Automation, $8(5)$, pp. 644-651.

[12] Yi, B., Freeman, R., and Tesar, D., 1994. "Force and stiffness transmission in redundantly actuated mechanisms: The case for a spherical shoulder mechanism". Robotics, Spatial Mechanisms, Mechanical Systems, 45, pp. 163-172.

[13] Kotlarski, J., Trung, D., Heimann, B., and Ortmaier, T., 2010. "Optimization strategies for additional actuators of kinematically redundant parallel kinematic machines". In Proceedings of the IEEE International Conference on Robotics and Automation (ICRA 2010).

[14] McAree, P. R., 1999. "An Explanation of NeverSpecial Assembly Changing Motions for 3-3 Parallel Manipulators". The International Journal of Robotics Research.

[15] Zein, M., Wenger, P., and Chablat, D., 2008. "Non-singular assembly-mode changing motions for 3-rpr parallel manipulators". Mechanism and Machine Theory, 43(4), pp. 480-490.

[16] Briot, S., and Arakelian, V., 2008. "Optimal force generation of parallel manipulators for passing through the singular positions". International Journal of Robotics Research, 27(8), pp. 967-983.

[17] Ider, S. K., 2005. "Inverse dynamics of parallel manipulators in the presence of drive singularities". Mechanism and Machine Theory, 40, pp. 33-44.

[18] Jui, C. K. K., and Sun, Q., 2005. "Path tracking of parallel manipulators in the presence of force singularity". ASME Journal of Dynamic Systems, Measurement and Control, 127, pp. 550 563.

[19] Khalil, W., and Dombre, E., 2002. Modeling, Identification and Control of Robots. Hermes Penton London.

[20] Khalil, W., and Guégan, S., 2002. "A novel solution for the dynamic modeling of gough-stewart manipulators". In Proceedings of the IEEE International Conference on Robotics and Automation (ICRA 2002).

[21] Müller, A., 2005. "Internal preload control of redundantly actuated parallel manipulators - its application to backlash avoiding control". IEEE Transactions on Robotics, 21(4), pp. 668-677.

[22] Gauthier, J.-F., Angeles, J., and Nokleby, S., 2008. "Optimization of a test trajectory for scara systems". Advances in Robot Kinematics: Analysis and Design. 
[23] Kanayama, Y., and Miyake, N., 1985. "Trajectory generation for mobile robots". In International Symposium on Robotics Research, pp. 333 -340 .

[24] Walton, D., and Meek, D., 2005. "A controlled clothoid spline". Computers and Graphics, 29(3).

[25] Meckl, P., and Arestides, P., 1998. "Optimized s-curve motion profiles for minimum residual vibration". In Proceedings of the American Control Conference.

[26] Wilde, D., 2009. "Computing clothoid segments for trajectory generation". Intelligent Robots and Systems, IROS 2009, pp. 2440-2445.

[27] Liu, X.-J., Wang, J., and Pritschow, G., 2006. "Kinematics, singularity and workspace of planar 5r symmetrical parallel mechanisms". Mechanism and Machine Theory, 44(2), pp. 145169. 